\title{
Erythema nodosum - presentation of three cases
}

\author{
Aleksandra Starba, Małgorzata Chowaniec, Piotr Wiland \\ Department of Rheumatology and Internal Diseases, University Hospital in Wroclaw, Wroclaw, Poland
}

\begin{abstract}
Erythema nodosum (EN) is a well-known form of panniculitis. It may occur as an idiopathic form and also can be induced by certain clinical conditions such as infections and granulomatous diseases such as sarcoidosis. Erythema nodosum may be accompanied by general symptoms such as fever, weakness, and severe pain, but skin lesions resolve without skin damage. Erythema nodosum occurrence is for the clinician a signal for a wider diagnosis and determination of the cause of occurrence of symptoms. The article presents three cases of EN with different causes and treatment.
\end{abstract}

Key words: erythema nodosum, infection, Crohn's disease, treatment.

\section{Introduction}

Erythema nodosum (EN) is a form of panniculitis, which is most common in women in middle age, and occurs particularly in spring, which is associated with streptococcal infections. In younger people EN occurs in correlation with increased incidence of sarcoidosis.

The picture of EN is characteristic: painful subcutaneous tumors, located typically on anterior sides predominantly of lower extremities. The changes resolve without skin ulcerations spontaneously within a few weeks (mean 2-8). Skin symptoms may be associated with arthralgia, fever, and weakness. The article presents 3 cases of erythema nodosum of various origins.

\section{Case 1}

A 27-year-old male patient, non-smoker, presented to the Rheumatology Department with a 3-week history of painful erythematous lesions on both shins. He also mentioned low-grade fever (up to $37.5^{\circ} \mathrm{C}$ ), fatigue and arthralgia. He did not have any significant past and family medical history. There was no history of a similar episode in the past. There were no symptoms of cough or weight loss and no history of any contact with tuberculosis. He did not take any medications. Physical examination revealed multiple rounded purplish nodules located bilat- erally on the extensor surface of the lower extremities. Tarsal joints were swollen and tender. Additionally, the patient was afebrile, in a good general condition (Fig. 1). Laboratory investigations revealed an elevated C-reactive protein - CRP (119.82 mg/l, normal $5.0 \mathrm{mg} / \mathrm{l}$ ) and erythrocyte sedimentation rate - ESR $(74 \mathrm{~mm} / \mathrm{h}$; normal 0-10 mm/h). Urine and blood culture results were negative. Throat swab revealed growth of normal flora. The diagnostic test for Yersinia was negative. His chest $X$-ray revealed bihilar lymphadenopathy. Further evaluation with high resolution chest computed tomography confirmed the lymphadenopathy and also demonstrat-

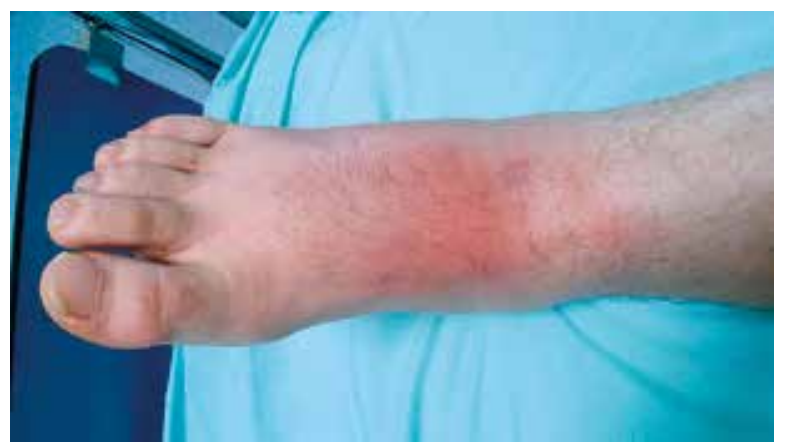

Fig. 1. 27-year-old male with acute stage of erythema nodosum. 
ed thickened bronchial walls of both lungs and nodular lesions, which suggested an alveolar sarcoidosis. The foot ultrasound showed a small amount of fluid in the right ankle joint and effusion in all sheaths of the flexor, extensor digitorum and the big toe tibial and peroneal tendons. Sonography also showed massive bilateral swelling of the subcutaneous tissue up to $1 / 2$ shank.

Due to nonspecific signs of infection (high serum level of (RP and ESR) the patient received empirical antibiotic therapy. Furthermore, the patient was referred for pulmonary consultation, where due to the high probability of sarcoidosis, prednisone treatment (40 $\mathrm{mg} / \mathrm{d})$ with a gradual reduction in dose was recommended (Fig. 2). After 2 days the skin lesions had completely resolved and after a month of treatment control chest $X$-ray showed regression of the lung alterations. The patient was referred to the Pulmonology Department, where based on the clinical and radiological examination the diagnosis of sarcoidosis was confirmed without histopathological examination.

\section{Case 2}

A 30-year-old female patient with no significant past medical history was admitted to the Rheumatology Department with complaints of arthralgia and painful nodular skin lesions on the bilateral lower and upper limbs

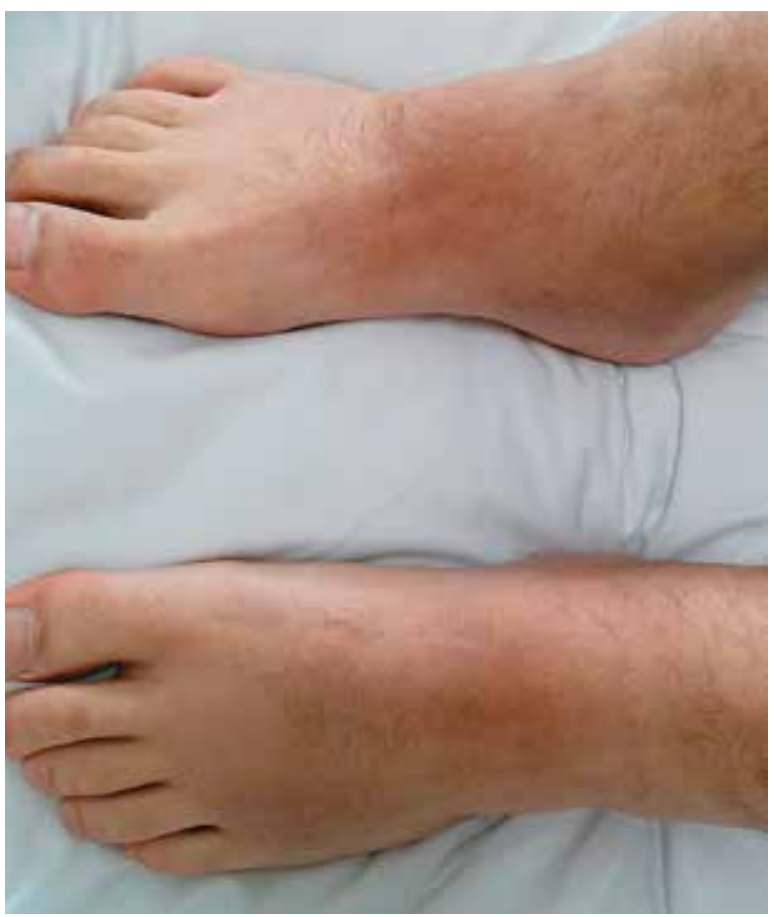

Fig. 2. Subsiding lesions after treatment with glicocorticosteroides, visible brown tinted postinflammatory hyperpigmentation. that developed one week earlier. These symptoms were accompanied by fatigue and fever up to $38^{\circ} \mathrm{C}$. The patient also reported sore throat that appeared $2-3$ weeks before admission to the hospital. Furthermore, the patient gave birth four months earlier. Both pregnancy and confinement proceeded without any complications.

Physical examination of the musculoskeletal system revealed swelling, pain and limitation of movement in the wrist, knee and ankle joints. Lower and upper limb examination showed multiple tender erythematous nodular skin lesions, $3-5 \mathrm{~cm}$ in diameter. The rest of the systemic examination was unremarkable.

Laboratory tests showed an elevated erythrocyte sedimentation rate $(94 \mathrm{~mm} / \mathrm{h}$; normal 0-10 mm/h), elevated C-reactive protein $(265.64 \mathrm{mg} / \mathrm{l}$, normal $5.0 \mathrm{mg} / \mathrm{l})$, leukocytosis $\left(13.32 \times 10^{9} / \mathrm{l}\right)$ and mild anemia (blood hemoglobin level of $11.6 \mathrm{~g} / \mathrm{dl}$ ). Urine and blood culture results were negative. Throat swab revealed growth of Klebsiella oxytoca, Moraxella catarrhalis and Streptococcus oralis. Urine and blood culture tests were negative. Chest imaging findings (chest X-ray and chest $\mathrm{CT}$ ) did not detect any lymphadenopathy or nodular lesions. Abdominal ultrasonography was within normal limits. The knee and foot ultrasound showed a small amount of synovial fluid.

Due to the history of upper respiratory tract infection 2-3 weeks previously, although the cultures of ordinary throat swab did not detect streptococcal infection, the patient received antibiotic therapy (mesalazine and azathioprine) and prednisone treatment $(20 \mathrm{mg} / \mathrm{d})$ with complete resolution of skin lesions.

\section{Case 3}

A 40-year-old man presented with a 1-week history of painful erythematous lesions on both shins. Past medical history revealed Crohn's disease diagnosed at age 33. Because of perianal fistula the patient required a colostomy which had been performed 3 weeks prior to hospitalization.

On admission the patient was afebrile. Physical examination revealed lesions clinically suggestive of erythema nodosum on the extensor parts of both lower legs and the right thigh. Examination of the musculoskeletal system revealed swollen and tender knee and ankle joints. A colostomy stoma was also visible.

Laboratory investigations showed an elevated erythrocyte sedimentation rate $(44 \mathrm{~mm} / \mathrm{h}$; normal $0-10$ $\mathrm{mm} / \mathrm{h}$ ), C-reactive protein ( $85.46 \mathrm{mg} / \mathrm{l}$, normal $5.0 \mathrm{mg} / \mathrm{l})$, mild anemia (blood hemoglobin level of $12.6 \mathrm{~g} / \mathrm{dl}$ ) and proteinuria $0.5 \mathrm{~g} / \mathrm{dl}$ (normal to $0.2 \mathrm{~g} / \mathrm{dl}$ ). Ultrasound of the knee, hand and foot showed a small amount of synovial fluid. 
The patient was given prednisone at a dose of $40 \mathrm{mg}$ per day and maintained therapy of mesalazine and azathioprine. He had a response with complete resolution skin lesions in a few days.

\section{Discussion}

Above we have presented the most common etiological factors of erythema nodosum. Patients were close in age, but the analysis of each case allowed the relationship of EN with a different cause, and according to this the therapeutic option was determined. The most common etiological factor of erythema nodosum is infectious agents, especially streptococcal [1].

Not very common, but also worth taking into account should be infections caused by Mycobacterium tuberculosis. Skin lesions appear usually 2-3 weeks after pharyngitis, which can be an important investigative clue. As we showed in the case above, antibiotic treatment associated with glucocorticosteroids is usually sufficient in this cases. Sarcoidosis is the second most common etiological factor of EN. When it occurs with arthritis and lung hilar lymphadenopathy it is called Löfgren syndrome. Usually it is a manifestation of the acute clinical form of the sarcoidosis, frequently occurring among young adults [2].

In each case of erythema nodosum at least a chest X-ray should be performed for assessment of the lung hilar nodules. The patient in the case described by us was diagnosed with sarcoidosis as Löfgren syndrome without histopathological examination. In this case, the criteria for Löfgren syndrome (bilateral hilar adenopathy, ankle arthritis, erythema nodosum and constitutional symptoms) were fulfilled. The good response to prednisone treatment and regression of lung alterations in control chest radiography also support the diagnosis of sarcoidosis. It is important to remember that hilar lymphadenopathy can also be observed in mycobacterial infections, as well as in more serious diagnoses such as lymphoma or leukemia, so this entity should be excluded before treatment administration [3].

Although inflammatory bowel diseases are not a very common cause of EN, the most common skin manifestation of inflammatory bowel diseases is erythema nodosum. It occurs in 4-15\% of patients with Crohn's diseases and $3-10 \%$ of patients with colitis ulcerosa. It is presumed that skin lesions of erythema nodosum correlate with the activity of bowel disease, and in patients with Crohn's disease, as in the case described by us, colonic involvement is observed more often [4]. Treatment of inflammatory bowel disease usually leads to resolution of skin lesions.
The presented cases show that erythema nodosum may be an entity with many underlying causes, and many clinical aspects should be considered in the diagnostic process. It is important and should result in selection of the appropriate therapy.

The authors declare no conflict of interest.

\section{References}

1. Sokolik R, Wiland P. Rumień guzowaty. In: Wielka Interna. Reumatologia. Puszczewicz M. (ed.). Medical Tribune Polska, Warszawa 2010; 226-230.

2. Grzelewska-Rzymowska I. Sarkoidoza - choroba ogólnoustrojowa. Alergia 2012; 2: 23-31.

3. Schwartz RA, Nervi SJ. Erythema nodosum: a sign of systemic disease. Am Fam Physician 2007; 75: 695-700.

4. Huang BL, Chandra S, Shih DQ. Skin Manifestations of inflammatory bowel disease. Front Physiol 2012; 3: 1-6. 